\title{
Patients' management of type 2 diabetes in Middle Eastern countries: review of studies
}

\author{
Zahra Khalil Alsairafi, ${ }^{1}$ Kevin Michael Geoffrey Taylor, ${ }^{2}$ Felicity J Smith, ${ }^{1}$ and Abdulnabi T Alattar ${ }^{3}$ \\ ucnvzka@ucl.ac.uk kevin.taylor@ucl.ac.uk, f.j.smith@ucl.ac.uk, abdul_2345@yahoo.com
}

${ }^{1}$ Research Department of Practice and Policy 2Department of Pharmaceutics, School of Pharmacy, University College London, UK ${ }^{3}$ Diabetes Unit, Al-Amiri Hospital, Ministry of Health, Kuwait

Abstract The increased prevalence of diabetes in Middle Eastern countries is a health policy priority. Important risk factors for diabetes have been identified. Lifestyle interventions and adherence to medications are central to disease prevention and management. This review focuses on the management of type 2 diabetes mellitus in Middle Eastern countries. The aim is to identify the ways in which knowledge, health beliefs, and social factors influence adherence to medication and lifestyle measures. Thirty-four studies were identified following a systematic search of literature. The studies describe the in influence of knowledge, health beliefs, culture, and lifestyle on the management of the disease in the region. Findings indicate a lack of health knowledge about diabetes, which has implications for health behaviors, medication adherence, and treatment outcomes. Many health beliefs and cultural lifestyle factors, such as religious beliefs, beliefs about fasting during Ramadan, and sedentary lifestyles played a role in patients' decisions. For better management of this disease, a collaborative approach between patients, their families, healthcare professionals, and governments should be adopted. Implementing behavioral strategies and psychological interventions that incorporate all healthcare professionals in the management process have been shown to be effective methods in changing patients' behavior. However, the utilization of such services and interventions is still limited in Arabian countries.

Keywords: type 2 diabetes mellitus, adherence, health beliefs, lifestyle, Ramadan, diet

\section{Introduction}

Diabetes mellitus (DM) is a leading cause of death in industrialized and developing countries. In the Middle East, DM is a rapidly growing chronic disease. Saudi Arabia, Kuwait, Qatar, and Egypt are among the ten countries in the world with the highest prevalence of DM. Major contributory factors for type 2 diabetes mellitus (T2DM), which is most prevalent in Middle Eastern countries, include low activity levels, poor diet, and excess body weight. Adherence refers to "the extent to which a person's behavior: taking medication, eating a suitable diet, and/or executing lifestyle changes correspond with agreed recommendations from a healthcare provider". Adherence was found to be low among Middle Eastern populations. In particular, studies have estimated medication non-adherence rates for diabetes to be $68 \%$ or lower. The main reasons for patients' non-adherence were forgetfulness, confusion about the required duration for using medication, and disbelief about the overall effectiveness of the medication.

This review aims to identify ways in which knowledge, health beliefs, and culture influence adherence to medication and lifestyle measures for T2DM patients in Middle Eastern countries, to outline all the determinants of patients' behavior to assist researchers to further investigate the issues identified or to identify new ones and to assist in identifying policy implications and propose recommendations to enhance the management of this disease in the region and improve health outcomes.

\section{Methods}

Search terms and search strategy- A systematic search of studies relating to medication adherence in diabetes in the Middle East was performed using the following databases: EMBASE, PubMed, Web of Science, and Index Medicus for the Eastern Mediterranean Region; the search was selected for the period from 1990 to 2015. The search terms were (diabetes AND adherence or compliance AND medicine use AND Middle East OR Kuwait OR United Arab Emirates OR Saudi Arabia OR Bahrain OR Qatar OR Oman OR Jordan OR Egypt OR Lebanon OR Syria OR Iraq OR Iran OR Palestine OR Israel OR Turkey OR Libya), with or without the combination of the search terms (health beliefs, lifestyle, patient behaviour, insulin). Studies that discussed medicine use during Ramadan were searched using the search terms (Ramadan fasting AND diabetes mellitus).

Inclusion/exclusion criteria- The electronic database search yielded 834 studies. Removing duplicates resulted in a total of 822 studies. Titles and abstracts were evaluated to determine eligibility for full screening. Studies that employed acceptable quantitative and/or qualitative methods and review articles were included. Studies wherein adherence to medicines and/or lifestyle among adults (18 years and older) with DM in Middle Eastern countries were explored were eligible for inclusion. Studies that involved all sexes and ethnicities, urban and rural, residents and expatriates, and all socioeconomic and educational backgrounds were included. Overall, 34 studies were classified as appropriate for full review. Eight studies were excluded due to unavailability of full access to the study ( $n=3)$, the quality of the study $(n=3)$ or due to inclusion of irrelevant subjects, e.g. pregnant women $(n=2)$. Examples of some of the included studies are shown in the following Table.

\begin{tabular}{|l|l|l|l|}
\hline \multicolumn{1}{|c|}{ Study setting } & \multicolumn{1}{|c|}{ Sample } & \multicolumn{1}{c|}{ Methods } & \multicolumn{1}{c|}{ Findings } \\
\hline $\begin{array}{l}\text { Al-Shookri et al, } \\
\text { Oman }\end{array}$ & $\begin{array}{l}\text { Patients with } \\
\text { T2DM }\end{array}$ & Review & $\begin{array}{l}\text { Lifestyle \& } \\
\text { behaviour of } \\
\text { patients } \\
\text { contribute to the } \\
\text { high prevalence } \\
\text { of T2DM }\end{array}$ \\
\hline $\begin{array}{l}\text { Salti et al, } \\
\text { Mediterranean } \\
\text { countries }\end{array}$ & $\begin{array}{l}\text { 12,243 patients } \\
\text { with DM }\end{array}$ & $\begin{array}{l}\text { Retrospective } \\
\text { survey. } \\
\text { Adherence of } \\
\text { patients to } \\
\text { medicines in } \\
\text { Ramadan }\end{array}$ & $\begin{array}{l}50 \% \text { of patients } \\
\text { changed their }\end{array}$ \\
$\begin{array}{l}\text { treatment doses } \\
\text { in Ramadan }\end{array}$ \\
\hline $\begin{array}{l}\text { Al-Qasem et al, } \\
\text { Mediterranean } \\
\text { countries }\end{array}$ & $\begin{array}{l}\text { Patients with } \\
\text { chronic diseases }\end{array}$ & Review & $\begin{array}{l}\text { Medication non- } \\
\text { adherence is } \\
\text { prevalent among } \\
\text { patients with DM }\end{array}$ \\
\hline
\end{tabular}

Results \& Discussion

The review revealed that lack of health awareness, health beliefs, and culture have a significant influence on patients' adherence to treatment and lifestyle measures. Different methodologies were employed to obtain the results of the reviewed studies. Most studies used questionnaires to collect data, whereas few studies applied interviews and focus groups. All the reviewed studies were conducted with adult patients with DM (predominantly T2DM). One study was conducted with participants at high risk of T2DM and two studies were conducted with health care professionals.

Lack of health awareness is common among patients with DM in the Middle East. It has been found that patients lacked an awareness of the seriousness of diabetes. In addition, this poor awareness was associated with different practices, such as the use of herbs instead of conventional treatment, and stopping or taking less medication to avoid adverse effects. Also, lack of health awareness of the role of diet and exercise led to difficulties in weight management and obesity. Some specific beliefs of the Middle Eastern population were also identified. Examples of are religious beliefs in God's control and in the "evil eye", beliefs about fasting in Ramadan, self-belief, and beliefs about necessity of treatment. From the studies that discussed these beliefs, it was conspicuous how these beliefs impacted patients' behavior and the management of diabetes. It was found that culture impacted adherence to lifestyle measures, such as diet and physical activity, and led to high obesity rates in the region and consequent poor health outcomes.

\section{Conclusion}

Prevention and management of T2DM is a priority for governments and people in Middle Eastern countries. This review identifies a lack of health knowledge about diabetes among populations. The lack of awareness impacts on health behaviors and medication adherence and has implications for treatment outcomes. Knowledge, beliefs, and cultural and lifestyle factors play an important role in patients' decisions regarding medication adherence and lifestyle interventions. 